\title{
Galileo's Gauge: Understanding the Empirical Significance of Gauge Symmetry
}

\author{
Nicholas J. Teh \\ Department of Philosophy \\ University of Notre Dame
}

August 27, 2015

\begin{abstract}
This article investigates and resolves the question of whether gauge symmetry can display analogs of the famous Galileo's ship scenario. In doing so, it builds on and clarifies the work of Greaves and Wallace (2014) on this subject.
\end{abstract}

\section{Introduction}

Philosophical reflection on the role and significance of physical symmetry is now in a mature and vigorous state of development. On the one hand, it incorporates problems that have emerged organically from a long tradition of thinking about symmetry within natural philosophy; on the other hand, the way in which we conceptualize these problems has been sharpened, transformed, and enriched by contemporary physics.

One such puzzle concerns how we should reconcile the seeming tension between the following two aspects of the symmetries of a physical theory, viz.

(Identical) The idea that a symmetry is a transformation between different representations of the same physical state of affairs.

(Empirical) The idea that symmetries have observable consequences, or more generally, empirical significance. They are thus supposed to 'guide' physicists in their construction of physical theories.

There is a well-known precedent for the discussion of this theme in natural philosophy, viz. the Galileo's ship thought experiment. The gist of it will be familiar: Salvatius imagines being inside an enclosed ship's cabin with various 'small flying animals' and claims that there will be no detectable change in their flight regardless of whether the ship is standing still, or in uniform motion. (Galileo, 1953) The thought experiment makes it plausible that if the laws of motion have Galilean symmetry, then boosting a stationary proper subsystem (the ship) of the universe into a state with some uniform velocity has no empirical consequences for measurements done within the subsystem 1 Nonetheless, it is possible for such a boost to bring about a physical difference in relation to the environment of

\footnotetext{
${ }^{1}$ That this is the case is itself an empirical fact, although not one that can be determined from within the subsystem.
} 
the subsystem: such a relational difference characterizes the empirical significance of symmetries in a Galileo's ship scenario.

The discussion of Galileo's ship assumes a new vitality when reconsidered in the light of the intricate and novel features of modern physical theories. Of these, gauge symmetry - by which we mean an internal, local symmetry (see Section 2.1 for the definitions) - stands out as a particularly interesting case, since it is the formal cornerstone of our most successful physical theories (e.g. the field theories of the Standard Model).

We thus turn to a brief review of 'Galileo's ship scenarios for gauge theory' in the philosophical literature. Kosso (2000) focuses on the empirical status of gauge symmetry. He notes that, on the one hand, a version of (Identical) always holds of symmetries: by definition, symmetry transformations leave certain features of the system (often taken to be the values of physical quantities) invariant. On the other hand, he notes that (Empirical) is true of various physical symmetries, i.e. one can directly observe a symmetry in the sense that (i) one observes that the transformation has taken place (with respect to a reference system), and (ii) one observes the invariance of the relevant features under that transformation. According to Section 4.4 of Kosso (2000), (i) holds of gauge symmetries, but (ii) does not - the invariance is not observed directly but requires positing a new force via the introduction of a gauge field.

Brading and Brown (2004) (BB) elaborate on Kosso's discussion and observe that a refinement of (i) and (ii) to subsystems - which captures the idea of the direct empirical significance (DES) of a symmetry (see Section 2.1 for the definition) - is the defining condition for Galileo's ship scenarios. However, they disagree with Kosso that (i) holds of gauge symmetry: by their lights, one cannot even observe that a gauge symmetry transformation has taken place. Thus, it is a fortiori the case that there are no Galileo's ship scenarios for gauge symmetry. Indeed, this claim has very much become part of the orthodoxy in the philosophy of physics (see e.g. Healey (2009); Budden (1997)).

This orthodoxy has recently been challenged by Greaves and Wallace (2014) (GW), who put the tension between (Identical) and (Empirical) in its starkest form, and aim to provide a conceptual framework for symmetries that reconciles the two aspects and shows the orthodox view to be false ${ }^{2}$ That is to say, they claim to vindicate the idea that gauge symmetry can indeed be used to manufacture Galileo's ship scenarios. On the face of it, this claim is indeed surprising, since it is conventional wisdom (in both the philosophy and physics communities) that gauge transformations are precisely those transformations that connect equivalent, and thereby redundant descriptions of the same phenomena - and how can the existence of these redundant descriptions have direct empirical consequences? ${ }^{3}$

In this paper, I will shed light on the above debate by refining, and building on, GW's work. First, although I endorse GW's positive proposal for using gauge symmetry to construct an analog of Galileo's ship, I will argue that some of their reasoning is flawed, because it is insufficient to show how Galileo's ship scenarios can arise in many cases of gauge theory.

Second, I will resolve what I call the logical puzzle about gauge, viz. how can gauge symmetries relate redundant descriptions and yet have direct empirical consequences? As we will see, the resolution of this puzzle lies in the fact that the term 'gauge transformation' is subtly ambiguous

\footnotetext{
${ }^{2} \mathrm{GW}$ do not use the language of 'observability', but they accept BB's criteria for a physical symmetry to have 'direct empirical significance'.

${ }^{3}$ Besides, the dialectical situation is yet more complicated: Friederich $(2014)$ has recently argued that, given what he takes to be plausible precisifications of GW's assumptions, one can actually deduce the negation of their claim. There is no room in this article to give a proper treatment of Friederich's argument, but it seems to me that GW can resist his conclusion on their own terms - I sketch a possible line of response in fn. 28.
} 
between two different notions, only one of which plays the role of connecting various redundant descriptions of a dynamical system.

Bringing these two points together helps us understand the precise sense in which one can (quite generically) use 'gauge symmetry' to construct Galileo's ship scenarios. Furthermore, it allow us to see why the infelicities present in GW's view inevitably evoke resistance from those (like BB) who defend the orthodox view of gauge symmetry. By making this friendly amendment to GW's view, I hope to achieve a rapprochement between it and the orthodox view, while preserving all of its insights.

\section{Background to the debate}

\subsection{Symmetries of physical theories}

In the contemporary debate, the symmetries of a physical theory are standardly taken to be transformations that act on the set of (mathematical) states of the theory, and which preserve the theory's laws of motion. This idea can be spelled out in various ways. Since our main examples will come from classical (Yang-Mills) gauge field theory, we will mostly adopt the standard picture of a symmetry acting on the space of fields, and leaving the theory's action invariant - this invariance ensures that such symmetries will also preserve the equations of motion (EOM), i.e. map field configurations that are solutions of the EOM to other such solutions ${ }^{4}$ One can also introduce an analysis of symmetries in terms of constraints, which classifies symmetries in various ways and in particular characterize the sense in which gauge symmetries relate redundant descriptions: it will be useful for us to apply this analysis in Section 4.1.

In order to proceed, we will need to recall the standard taxonomy of symmetries in modern physics. This is usually broken into two logically independent distinctions. So a symmetry will generally be described by two predicates: one from each pair.

First, the external (also called space-time) v. internal symmetry distinction. External symmetries are those transformations that effect a change in the space-time parameters (e.g. $x, y, z, t)$ of a theory. On the other hand, transformations that do not affect the space-time parameters are said to change only 'internal parameters' and are thus called the internal symmetries of a theory ${ }^{5}$

Next, the global v. local symmetry distinction. Very roughly speaking, global symmetries are transformations that do not depend on space-time parameters, whereas local symmetries are functions from space-time parameters to a 'gauge group' $G$ of transformations, e.g ${ }^{6} g: \mathbb{R}^{3,1} \rightarrow G$, where $\mathbb{R}^{3,1}$ is 4-dimensional Minkowski space-time.

This rough (albeit pervasive, in both the physics and philosophy literature) characterization in fact obscures a subtle and important point about the global vs. local distinction, which will turn out

\footnotetext{
${ }^{4}$ Here I set aside many subtleties concerning the characterization of symmetries. See e.g. Belot (2011), who discusses the problems with drawing too tight a connection between various formal notions of symmetry and the notion of physical equivalence between states - these will not concern us as the problematic that I will consider arises even with the most naive construal of such a connection. For a canonical exposition of the idea that the invariance of laws implies the invariance of equations of motion, see Olver (1993).

${ }^{5}$ One can also construct theories with supersymmetry, which is sometimes said to relate space-time and internal symmetries, but this is only true insofar as one considers the classical correlates of fermionic degrees of freedom (i.e. Grassmann coordinates, which form part of superspace) to be 'internal parameters'.

${ }^{6}$ In fact, the distinction is not merely binary: one can actually formulate a whole series of intermediate types of symmetries between global and local, see e.g. Nussinov et al. (2006).
} 
to be a source of philosophical confusion in the debate. I thus give a more careful characterization of this distinction at the end of Section 2.2, in the context of gauge theories.

Following standard practice, we shall say that a global internal symmetry transformation $g \in G$ (where $G$ is some Lie group) is localized when we turn $g$ into a function $g(x)$ from space-time to $G$, i.e. it is a gauge transformation in the general sense. Physicists often speak of 'localizing' a symmetry as a method of moving from a theory with a global symmetry to a theory with a corresponding local symmetry. On the other hand, we shall refer to the reverse procedure - replacing functions into $G$ with constant elements of $G$ - as the 'globalizing' of a gauge symmetry.

Three caveats must be issued about the use of 'local' and 'gauge' in this paper. First, some authors use the terms 'local symmetry' and 'gauge symmetry' synonymously $]^{7}$ However, in keeping with the most popular use of the term in physics, we will take gauge symmetry to refer only to the internal, local symmetries of Yang-Mills type theories, which we review in Section 2.2.

The second caveat is of greater significance. Even after restricting 'gauge symmetry' to the internal local symmetries of Yang-Mills theory, the use of this notion in much of the philosophical/physics literature is ambiguous between two different ideas: First, the general and formal notion that we have just been using, viz.

(Formal) The group of gauge transformations $\mathcal{G}$ is the set of maps from space-time to $G$ (under whose action fields transform in the appropriate way - see Section 2.2 for details of the Yang-Mills case) ${ }^{9}$

And second, we can further demand that the functional role of 'representing redundancy' is satisfied by (Formal) gauge transformations, i.e.

(Redundant) A necessary condition on gauge transformations is that they connect different descriptions of the same physical state of affairs, where a 'description' is taken to be a (mathematical) state of a well-defined dynamical system (viz. where we have fixed the type of solution of interest, the initial conditions, and the boundary conditions).

It is easy to slide between (Formal) and (Redundant) gauge transformations, or simply identify them, in casual conversation. But there is no reason to expect these concepts to align in general. On the one hand, satisfying the functional role of redundancy is clearly not a sufficient condition for being a gauge transformation since - as we note below - global symmetries of the universe also satisfy the functional role of representing redundancy. On the other hand, we will see in Section 4 that not all (Formal) gauge transformations are (Redundant).

Third, note that the term 'local' is also used in a different sense in discussions of geometry and topology. To disambiguate, we shall use 'local' to refer to properties that can be predicated of an arbitrarily small, and in particular topologically trivial, patch of a smooth manifold. By contrast, global properties are those that hold of an entire (possibly topologically non-trivial) manifold.

\footnotetext{
${ }^{7}$ To complicate matters, some authors use the term 'gauge symmetry' to include any symmetry that connects redundant descriptions of a state of affairs, so that global symmetries of the universe will count as gauge symmetrieswe will not adopt this usage.

${ }^{8}$ The points made in this paper hold for local symmetry generally, regardless of whether it is internal or external. However, we focus on the Yang-Mills case-where the local symmetries are internal-for concreteness and ease of illustration.

${ }^{9}$ The composition operation for two such maps $g(x), h(x) \in G$ is just $g(x) \cdot h(x)$ where $\cdot$ is the composition operation of the group $G$.
} 
Many (though not all) agree that, regardless of the type of a symmetry, it exhibits (Identical) when it acts on the entire universe 10 After all, what physical difference can it make to simultaneously transform everything in the universe in the same way? There is also a consensus that, when an external global symmetry acts on a proper subsystem of the universe, then it can in principle exhibit (Empirical), just as in the case of Galileo's Ship 11 This is because such a transformation can effect a (genuine, physical) relational difference between the subsystem and its environment and thus a genuine difference between the pre- and post-transformation states of the universe.

However, apart from this, controversies about (Empirical) abound ${ }^{12}$ This paper's focus is a specific controversy about whether gauge symmetries can have direct empirical significance: GW claim that this is possible, but their claim is denied by Kosso, Brading and Brown (BB), and Healey (and many others), who thus represent the orthodoxy in this debate.

Let us now recall how the terms direct and indirect empirical significance have been used in this literature:

Direct Empirical Significance (DES) ${ }^{13}$ A symmetry acting on a dynamically isolated proper subsystem (of the universe) has DES when the transformed and untransformed subsystems are (i) empirically indistinguishable from within the subsystem; but (ii) empirically distinguishable with respect to a reference/environment system (i.e. the 'rest of the universe', in an appropriate sense).

Indirect Empirical Significance (IES): A symmetry has IES when it is possible to empirically verify the consequences of the symmetry. The primary examples of such consequences are given by Noether's theorems, e.g. Noether's first theorem shows that there is a conservation law (for a 'Noether charge') associated with each global symmetry, and her second and third theorems show that a local symmetry imposes very strong restrictions on the possible form of a physical theory ${ }^{14}$

DES is formulated in such a way as to capture the sense of (Empirical) that is characteristic of Galileo's ship scenarios. IES, on the other hand, covers a far wider range of possible implications of a physical symmetry under its rubric of 'indirect'. For us, the most important implication of IES will be the idea that a charge is associated with each global symmetry via Noether's theorem. A charge in this highly general sense is some attribute of a dynamical object (e.g. a field) that determines how this object will respond to some particular force. Indeed, each charge is typically 'conjugate' to some force and serves to characterize the force, as well as to provide a constant conserved over time by the dynamics. This aspect of IES is closely related to DES: in a Galileo's ship scenario, the relational difference between the transformed subsystem and its environment is precisely a relative difference in a generalized charge of this kind. Thus, in order to effect such a scenario, a subsystem symmetry must give rise to a charge that is well-defined and conserved.

\footnotetext{
${ }^{10}$ Some will protest that a universe symmetry only relates empirically indistinguishable states, which are nonetheless physically distinct. Fair enough - but this metaphysical contention will not affect either my or GW's discussion below, because the notion of 'physical difference' that concerns us is the empirical distinguishability of a transformed subsystem relative to an environment system (see the definition of DES below).

${ }^{11} \mathrm{Cf}$. the relational sense discussed on p. 2.

${ }^{12}$ Historically, part of this controversy has been framed in terms of 'observability'. For instance, Kosso claims that both global and local internal symmetries are observable, but BB deny this. We shall not be concerned with these aspects of the debate, and will put aside the idea of 'observability' in favor of 'direct empirical significance'.

${ }^{13}$ As first articulated in Brown and Sypel (1995), and then discussed by Kosso (2000) and BB.

${ }^{14}$ See Section 4.4 of Olver (1993) for a technical discussion of Noether's first theorem, and Brading and Brown (2000) for a philosophical discussion of Noether's theorems in relation to gauge symmetry.
} 
While some parts of the debate have been conducted at a rather high, i.e. non-theory-specific, level of generality (see e.g. Greaves and Wallace (2014) and Kosso (2000)), much can be learned by exploring these issues in the case of one large and particularly important class of theories, viz. YangMills (often referred to simply as: gauge) theories. The reasons are several. First, field theories - and in particular gauge theories - are radically different from particle theories, not least because they allow for non-trivial long-range interactions: as we will see in the case of gauge fields. Second, gauge theories have highly complex symmetry transformations (viz. maps into non-Abelian Lie groups) which allows us to appreciate the richness and novelty of local symmetry, while still being more tractable than the local symmetry of general relativity. Third, gauge theories are hardly speculative or inessential physics: they account for three of the four fundamental forces with which we have discovered. At any rate, the examination of whether Yang-Mills gauge symmetries can display DES dovetails neatly with one of GW's four main examples, i.e. a scalar field coupled to a $U(1)$ gauge field.

\subsection{Gauge theory}

Yang-Mills gauge theory, like any other field theory, has fields - i.e. maps from space-time to some abstract value space - as its dynamical variables. In the sketch that follows, we will be concerned with the local aspects of gauge theory (i.e. its properties on a small topologically-trivial patch $U$ of a space-time $M$ ); except at the end of Section 2.2, where I explain one important global fact about gauge theory.

Physicists often speak of the 'gauge group' of a theory - let us stress that this concept is not to be confused with 'the group of gauge transformations'; it instead refers to the group (usually a Lie group such as $S U(n)$ or $S O(n)$ ) in which the gauge transformations take values. Locally, a

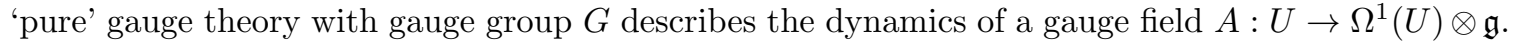
In other words, $A$ is a map from a patch of space-time $U$ to an abstract value space of $\mathfrak{g}$-valued 1 -forms 15

The gauge symmetry transformations of a gauge theory are usually defined locally, in a way that exemplifies (Formal) of Section 2.1. They are maps $g$ from space-time into the gauge group $G$, which act on a gauge field in the following manner ${ }^{16}$

$$
A_{\mu} \mapsto A_{\mu}^{\prime}=g A_{\mu} g^{-1}-\left(\partial_{\mu} g\right) g^{-1},
$$

where $\mu$ is a spacetime index. This symmetry is local (since $g$ is space-time dependent) and internal (since $g$ does not act on space-time parameters). Furthermore, it is clear that at this local level, the global transformations are a special case (i.e. just the constant functions) of the gauge transformations.

By extending the notion of a derivative to a gauge covariant derivative, we can define the field strength or curvature field $F$ : (in coordinates) $F_{\mu \nu}:=\partial_{\mu} A_{\nu}-\partial_{\nu} A_{\mu}+\left[A_{\mu}, A_{\nu}\right] . F$ is often taken to be the 'physical field' in elementary treatments of $U(1)$ gauge theory. However, various experimental and theoretical arguments (e.g. the Aharonov-Bohm effect) have shown that the physical degrees of freedom of a gauge theory are rather more subtle, and should instead be identified with various

\footnotetext{
${ }^{15}$ The space of 1 -forms is dual to the tangent space of the manifold (thus the relation to space-time) and the Lie algebra $\mathfrak{g}$ is the isomorphic to the tangent space at the identity of the Lie group $G$.

${ }^{16}$ This description suffices for our purposes, although note that there is an abstract invariant formulation of gauge transformations as the vertical automorphisms of a principal bundle.
} 
topological classes or objects (e.g. Wilson loops) that can be associated with the gauge field $A$. Thus, despite the representational redundancies of the $A$ field, physicists tend to treat it as the fundamental dynamical field variable of gauge theory.

We now proceed to dynamics. The curvature $F$ enters directly into the description of the dynamics of the theory, which is specified by the action $S=\operatorname{Tr}\left(\int F \wedge * F\right)$, where $\operatorname{Tr}$ is a non-degenerate inner product on the Lie algebra $\mathfrak{g}$ of the gauge group $G$. The theory's equations of motion can be derived from this action. Furthermore, the action is left invariant by gauge transformations, which ensures that such transformations will map a solution of the theory to another solution ${ }^{17}$

The main examples in this paper come not from pure gauge theory, but from coupling a gauge field to some other field. For instance, GW's example of a Galileo's ship scenario for gauge theory comes from a model of this sort (which they call Klein-Gordon-Maxwell (KGM) electrodynamics), cf. Section 3.2. In Section 4 we will consider a closely related model called Yang-Mills-Higgs (YMH) theory: this extension is obtained by coupling a scalar field $\phi$ to the gauge field $A$ and writing down an appropriate action to specify the dynamics of the theory.

The YMH action is $S=\operatorname{Tr} \int-\frac{1}{4} F_{\mu \nu} F^{\mu \nu}-\frac{1}{2} D_{\mu} \phi D^{\mu} \phi-V(\phi)$. As such, this theory has two interacting dynamical variables, viz. the fields $A_{\mu}$ and $\phi$, on which gauge transformations act. We will not need the detailed form of the potential $V(\phi)$, apart from noting that it is invariant under gauge transformations, and that its minimum energy submanifold (called the vacuum manifold) in the space of fields $\phi$ has the topology of a sphere $S^{2}$.

Our primary reason for introducing YMH theory is that in addition to linearized solutions about the vacuum manifold, it also contains a special class of topological soliton solutions called 'monopoles'. Like other topological solitons, monopoles are defined in terms of topologically nontrivial properties of a field configuration, which also accounts for their particle-like properties. We will see that such solutions have interesting implications for the question of whether gauge symmetry exhibits DES.

Finally, let us touch in a preliminary way on the relationship between global transformations and gauge transformations.

First recall that global symmetries play an important role in specifying the empirical content of a field theory, because they (via Noether's first theorem) give rise to a conserved current, which can in turn be integrated over a spatial slice to give rise to a conserved charge. In Section 2.1's taxonomy of 'empirical significance', this is classified as indirect empirical significance (IES); however, it is not any less important for that. Indeed, if one hopes to construct an instance of a Galileo's ship scenario (i.e. exhibit DES) for such theories, then one needs the notion of a relative difference in charge or phase between a subsystem and its environment. Furthermore, one needs such charges to be conserved, on pain of introducing unpredictable irregularities in the dynamics of charged objects.

How does this connection between symmetries and charges look when we move to a general gauge theory? As discussed in Brading and Brown (2000), one can still define conserved currents for a gauge theory (albeit with some novel aspects to the derivation). However, when one tries to integrate such currents over a spatial slice in order to obtain a charge, one sees that either (i) the spatial slice has no boundary and the charges vanish identically; or (ii) the spatial slice does have a boundary and the charges are ill-defined, because there is an arbitrariness in the object that one is integrating over the boundary 18

\footnotetext{
${ }^{17}$ The solutions of 'pure' $S U(n)$ classical Yang-Mills theory will be waves: linear waves in the electromagnetic case where $n=1$, and non-linear waves in the case where $n \geq 2$.

${ }^{18}$ In the case of $U(1)$ gauge theory, this arbitrariness can be fixed by just stipulating that the boundary object i.e. the superpotential - is proportional to $F_{\mu \nu}$ (because this makes the current vanish on-shell outside sources), but
} 
The moral of this observation is that in order for gauge theories to have a non-trivial well-defined charge, one needs to specify appropriate asymptotic boundary conditions. This was first recognized in an informal way in Abbott and Deser (1982) (where the existence of charges for Yang-Mills theory is related to boundary conditions with certain symmetries), but the study of how boundary conditions give rise to asymptotic charges has become a deep and technical subject (see e.g. Barnich and Brandt (2002) for a review). Thus one sees that unlike global symmetries, this aspect of the empirical content of a gauge theory depends on boundary conditions in complex and subtle ways.

The foregoing results should already give one pause about drawing too tight a connection between the empirical content of a global theory, on the one hand, and a gauge theory, on the other hand. For they tell us that, even if DES-exhibiting symmetries can be constructed from gauge symmetry, they will in general require the presence of special boundary conditions (i.e. those that allow one to construct a well-defined charge). In Section 4, we will see that precisely because of such conditions, the DES-exhibiting symmetries that emerge need not be the globalization of the gauge symmetry.

A yet more dramatic rift between global and gauge symmetry appears if one is prepared to consider scenarios where a spacetime $M$ has a nontrivial global topology: we see that the globalization of a gauge symmetry is not in general well-defined. Let us pause to explain this point.

In this general scenario, one needs to consider gauge transformations $\gamma_{i}: U_{i} \rightarrow G$, where the $U_{i}$ are different (possibly intersecting) topologically trivial patches that form a cover of the space-time $M$. Suppose $i=1,2$. Then the relationship between $\gamma_{1}$ and $\gamma_{2}$ on a non-empty intersection $U_{1} \cap U_{2}$ is given by:

$$
\gamma_{2}(x)=g_{12}^{-1}(x) \cdot \gamma_{1}(x) \cdot g_{12}(x),
$$

where $g_{12}$ is a transition function from $U_{1} \cap U_{2}$ to $G$. Such transition functions will be non-trivial (i.e. different from the identity) when $M$ is topologically non-trivial, and when combined with the fact that $G$ can be non-Abelian, this implies that the globalization of a gauge symmetry (i.e. setting $\gamma_{1}=\gamma_{2}=$ constant) will not exist in general. More precisely, for an arbitrary gauge group $G$, (2) can only be satisfied, and the globalization exist, when at least one of the following conditions is met: (a) space-time is topologically trivial (i.e. only one contractible patch $U_{i}$ is required to describe it)); and (b) when the gauge group is Abelian, e.g. $U(1)$.

The case of non-Abelian gauge theories on topologically non-trivial spacetimes is important because it helps us appreciate just how fundamentally different gauge symmetry is from global symmetry. But there are other ways in which topology can be physically significant, e.g. one can consider a topologically non-trivial boundary of a subsystem (which in turn has implications for the system's boundary conditions) which is accessible to an observer outside the subsystem. The morals of such subsystems for our topic will be explored in Appendix A.

\section{Can a gauge symmetry have direct empirical significance?}

GW's target is the claim, discussed in Section 1, that (i) only subsystem transformations can have DES, and thus (ii) global, but not gauge symmetries can have DES. They thus develop a framework that underwrites (i), and use it to show that - pace (ii) and the orthodoxy - the relevant distinction for distinguishing between symmetries that do and do not have DES is not the global/gauge distinction, but instead another distinction between what they call 'interior' and 'non-interior' symmetries.

no such quick fix is available for non-Abelian gauge theory. 
The reason for introducing this new distinction is that GW take (Identical) - or lack of DES to hold good only for symmetries of the universe, whose transformations cannot produce physically distinguishable states. Thus, interior symmetries - i.e. the ones that do not have DES - are roughly speaking defined as those subsystem symmetries which are also symmetries of the universe. $\mathrm{GW}$ are then concerned to argue that (ii) is false because some gauge symmetries are non-interior. Indeed, they claim that the gauge/global distinction is only related to the interior/non-interior distinction insofar as "only when the group of theoretical symmetries is "local" will there be any interior symmetries, distinct from the identity, for subsystems that are identifiable as subregions of space-time' (Greaves and Wallace, 2014, p. 28).

Thus, on GW's view, the main difference between gauge symmetries and global symmetries is independent of the issue of DES: it is that gauge symmetries give rise to interior symmetries with a far richer structure than global symmetries. Furthermore, certain pairs of gauge and global symmetries are supposed to be very similar with respect to DES: GW hold that the localization of a global theory with a DES-exhibiting symmetry gives rise to a gauge theory with a DES-exhibiting asymptotic version of the original global symmetry 19 I challenge this further claim in Sections 4.2 and Appendix A.

Notice that GW do not disambiguate their use of 'gauge transformation' between what I have called (Formal) and (Redundant). If one were to exclusively consider gauge transformations in the sense of (Redundant), then it seems logically impossible that these could exhibit DES. Thus, if they are right that gauge symmetry transformations can exhibit DES, we would have to conclude that these are only gauge transformations in the (Formal), but not the (Redundant), sense. This implication will be considered in Section 4; in this section, we focus on GW's argument for their view.

GW's argument proceeds in the following manner. First they present objections to the orthodox view based on the idea that global symmetries are contained in local symmetries. These objections are used to motivate a conceptual framework that allows them to characterize Galileo's ship scenarios in terms of certain abstract properties of symmetries (non-interiority and boundary-preserving-ness); they then show that there is a gauge theory example $(U(1) \mathrm{KGM})$ which meets this criterion. I endorse GW's claim that $U(1)$ KGM can provide a Galileo's ship scenario. However, the $U(1)$ case is most certainly not typical - one can think of it as a degenerate point in the space of gauge theories - and since GW's motivations/explanations for DES depend on special features of the $U(1)$ case, they fail to explain DES for generic gauge symmetries. The true explanation comes analyzing the logical puzzle about gauge, as I shall explain in Section 4.

\subsection{GW's objections to the orthodoxy}

GW offer three objections to the claim that gauge symmetries cannot have DES, which in turn provide a motivation for their positive proposal to construct analogs of Galileo's ship within gauge theory. I shall now criticize two of these objections (which I do not take to be crucial to their positive proposal) in order to refine and develop their position.

The first objection is based on the mantra that 'global symmetry is contained in (or is a special case of) local symmetry' - call this (GlobalInLocal) (cf. (Greaves and Wallace, 2014, p. 3)). Thus if global symmetries can have DES, so too can gauge symmetries.

\footnotetext{
${ }^{19}$ "Every "global" theory and the corresponding "local" theory will entail exactly the same empirical symmetries, with constant transformations in the former case replaced by equivalence classes of transformations in the latter' (Greaves and Wallace, 2014 p. 30).
} 
But as we have seen at the end of Section 2.2, the mantra is in general false. (GlobalInLocal) fails when one carefully considers the concept of a local - specifically gauge - symmetry transformation, because such transformations are in general required to satisfy a global consistency condition, i.e. (2). Is it then in any sense true that global symmetries are contained in local symmetries? As discussed in Section 2.2, this is true at the level of local symmetry transformations. Thus, in order for GW's objection to work, they would have to restrict it to the limited class of theories for which only local symmetry is relevant ${ }^{20}$ Furthermore, we should bear in mind that such local transformations (and the associated conserved currents) will not in general be sufficient for constructing Galileo's ship scenarios: what is needed for the latter is a relative difference in charge and the definition of such a charge in gauge theory depends crucially on the boundary conditions of the system, including the possibly non-trivial topology of a subsystem boundary.

GW's second objection is very closely related to the first. They suggest that (LocalizeEmpirical): the localization of a global theory should have the same DES-exhibiting symmetry group as the global theory, because this explains the 'preservation of empirical content' in the localization procedure. As we shall see, this is true of the $U(1)$ case, but generically false for gauge theory ${ }^{21}$

\subsection{GW's framework}

We now review GW's proposed framework for analyzing symmetries, and in particular for thinking about the abstract conditions under which Galileo's ship scenarios can arise. I adapt some of their notation and introduce the framework informally (for an axiomatization, see the final section of Greaves and Wallace (2014)). Throughout this section, 'gauge transformation' will be used in a (Formal) sense, in keeping with GW's usage (cf. the beginning of Section 3) - we will consider its relation to (Redundant) in Section 4.

Let $\mathcal{U}$ be the set of dynamically (including kinematically $\sqrt{22}$ possible states of the universe, also known as the total system, with a set of universe symmetries $\Sigma_{\mathcal{U}}$ acting on it. So for instance, in the case of Yang-Mills-Higgs (YMH) fields over the entire universe, $\mathcal{U}$ is the configuration space of fields $\mathcal{C}:=\{(A, \phi)\}$ on a space-time $M$ (which represents the universe), and $\Sigma_{\mathcal{U}}$ is the group of gauge symmetries $\mathcal{G}$ acting on $\mathcal{C}$.

The cases of interest to us are ones in which $\mathcal{U}$ can be decomposed into two sets of states: (i) a subsystem $\mathcal{S}$, which is dynamically isolated from (ii) its environment $\mathcal{E}$. In general, an arbitrary element of the Cartesian product $\mathcal{S} \times \mathcal{E}$ will not be 'physical' because the pair $\langle s, e\rangle$ (where $s \in \mathcal{S}$ and $e \in \mathcal{E}$ ) might not be compatible, i.e. they might not satisfy the right boundary conditions to form a dynamically possible state of the universe. GW assume that $s$ and $e$ are dynamically compatible just in case there is a universe state $u \in \mathfrak{U} \subset \mathcal{S} \times \mathcal{E}$ that projects onto these two factor states respectively. We can thus introduce the notation $u \equiv s \sqcup e$ to indicate that $u$ is the composite, or conjunction, of states from $\mathcal{S}$ and $\mathcal{E}$ respectively.

In GW's framework, one starts with a primitive set of universe symmetries $\Sigma_{\mathcal{U}}$ and uses restrictions of these to $\mathcal{S}$ and $\mathcal{E}$ to define their respective sets of subsystem symmetries $\Sigma_{\mathcal{S}}$ and $\Sigma_{\mathcal{E}}$. In

\footnotetext{
${ }^{20}$ Running the argument with this restriction does succeed in showing that some gauge symmetries can exhibit DES. However, fixating on this special case leads one to ignore the many more interesting ways in which gauge symmetry can exhibit DES.

${ }^{21} \mathrm{GW}$ are right that progress is often made by localizing a theory with a global symmetry; but such progress does not consist in 'retaining' the DES of the original global symmetry.

${ }^{22}$ The terminology of 'dynamical possibility' is from GW, but it is clear that they mean this to include kinematical possibility. In particular, the examples below rest on the kinematical impossibility stemming from discontinuities in field values at a geometric boundary.
} 
particular, we shall require that for all $s \in \mathcal{S}$ and $e \in \mathcal{E}$, a universe symmetry $\sigma \in \Sigma_{U}$ acting on the universe state $s \sqcup e=u \in \mathcal{U}$ restricts to subsystems in the following manner

$$
\sigma(s \sqcup e)=\left.\left.\sigma\right|_{\mathcal{S}}(s) \sqcup \sigma\right|_{\mathcal{E}}(e),
$$

and thus defines the subsystem symmetries $\left.\sigma\right|_{\mathcal{S}}=: \sigma_{\mathcal{S}} \in \Sigma_{\mathcal{S}}$ and $\left.\sigma\right|_{\mathcal{E}}=: \sigma_{\mathcal{E}} \in \Sigma_{\mathcal{E}}$.

Following GW, we also define the composition of two subsystem symmetries $k_{\mathcal{S}} \in \Sigma_{\mathcal{S}}$ and $l_{\mathcal{E}} \in \Sigma_{\mathcal{E}}$, i.e. for all $s \in \mathcal{S}$ and $e \in \mathcal{E}$,

$$
k_{\mathcal{S}} \sqcup l_{\mathcal{E}}(s \sqcup e):=k_{\mathcal{S}}(s) \sqcup l_{\mathcal{S}}(e),
$$

where we assume that the RHS is a well-defined state of the universe. Notice a very important feature of this definition: the composition of two subsystem symmetries need not be a universe symmetry! In other words, $k_{\mathcal{S}} \sqcup l_{\mathcal{E}}: s \sqcup e \mapsto k_{\mathcal{S}}(s) \sqcup l_{\mathcal{E}}(e)$ might be an automorphism of $\mathcal{U}$ that is not an element of $\Sigma_{\mathfrak{u}}$. The importance of this feature emerges in GW's gloss on the definition of DES: Recall that a universe symmetry $\sigma$ cannot effect a real physical difference; thus, a subsystem symmetry $\sigma_{\mathcal{S}}:=\left.\sigma\right|_{\mathcal{S}}$ can only exhibit DES if it is the restriction of a universe map which is not a universe symmetry.

Let us now illustrate how the subsystems $\mathcal{S}$ and $\mathcal{E}$ are defined in gauge theory. As GW note, in the case of field theories, a dynamically isolated subsystem can sometimes be defined by the restriction of fields (satisfying certain conditions) to some compact region of space or space-time ${ }^{23}$ So for instance, $\mathcal{S}$ might be explicitly defined as field configurations on a compact region $S$ within a space-time $M$; the environment system $\mathcal{E}$ is then defined as field configurations on $M-S$. Subsystem symmetries are then defined as the (spatio-temporal) restriction of the group of gauge symmetries $\Sigma_{\mathfrak{u}} \equiv \mathcal{G}$ on the entire space-time $M$, to $S$ and to $E$ respectively.

However, a crucial point in what follows will be that not all field-theoretic subsystems can be described in this way. Indeed, some field subsystems $\mathcal{S}$ have asymptotic properties that are highly non-trivial, a perspicuous description of which requires us to represent the boundary $\partial S$ of a spatial region $S$ as being located out at 'spatial infinity'. Of course, the use of 'infinity' here is merely an idealization: it does not mean that $\partial S$ is literally infinitely far away from the interior of $S$, but rather that, relative to some scale set by the physics, $\partial S$ is very far away from the 'core' of the field configuration. Nonetheless, the use of such infinities allows one to deploy a powerful mathematical description of the asymptotics of such field configurations. The point is that the formal description of some field subsystems $\mathcal{S}$ will not fit the simple model invoked by GW, namely the restriction of a field configuration to some compact region of space-time.

Let us now discuss the topic of boundary conditions of a state. We will need to clarify a terminological point: GW use 'the boundary condition $B_{s}$ of the state $s \in \mathcal{S}$ ' to refer to the set of all $e \in \mathcal{E}$, such that $s \sqcup e$ is dynamically possible, in the sense that the factor states are dynamically compatible; similarly, given $e \in \mathcal{E}, C_{e}$ denotes the set of all $s \in \mathcal{S}$ such that $s \sqcup e$ is dynamically possible. However, in field theory it is much more common to have the boundary conditions be (roughly) the - perhaps asymptotic - field values on the geometric boundary $\partial S$ meshing with $S$, and not the equivalence class of all $e \in \mathcal{E}$ that mesh with $s$. For us, the most important case will be when a field $\phi$ on three-dimensional space takes certain asymptotic values on the 'sphere at infinity', denoted $S_{\infty}^{2}$, of this space. We shall use the notation $\phi_{\infty}$ to represent the asymptotic values of such fields, i.e. the values they take on $S_{\infty}^{2}$. Such asymptotic values will of course determine boundary

\footnotetext{
${ }^{23}$ As GW note, however, in other physical theories it may not be possible to think of subsystems as defined by regions of space-time.
} 
conditions in GW's sense; however, they are not defined in terms of the $\mathcal{E}$ states that are compatible with a given $\mathcal{S}$ state.

We now turn to GW's basic taxonomy of symmetries of the subsystem $\mathcal{S}$. They introduce two (logically independent) types:

- An interior symmetry $\sigma_{\mathcal{S}}: \mathcal{S} \rightarrow \mathcal{S}$ is one such that, for all $s \in \mathcal{S}$ and $e \in \mathcal{E}$ such that $s \sqcup e$ is defined,

$$
\sigma_{\mathcal{S}} \sqcup 1_{\mathcal{\varepsilon}}: s \sqcup e \mapsto \sigma_{\mathcal{S}}(s) \sqcup e
$$

is also a universe symmetry, where $1_{\mathcal{E}}$ is the identity on $\mathcal{E}$. This condition implies that $\sigma_{\mathcal{S}}(s) \sqcup e$ is dynamically possible.

- A boundary-preserving symmetry $\sigma_{\mathcal{S}}: \mathcal{S} \rightarrow \mathcal{S}$ of a state $s$ is a subsystem symmetry such that for all $e \in \mathcal{E}$ compatible with $s, s$ and $\sigma_{\mathcal{S}}(s)$ are elements of the boundary condition $C_{e}$.

According to GW, interior symmetries are precisely those symmetries that cannot exhibit DES. They reason as follows: By definition, the interior symmetry $\sigma_{\mathcal{S}}$ acting on the subsystem $\mathcal{S}$ is equivalent to letting $\sigma_{\mathcal{S}} \sqcup 1_{\mathcal{E}}$ act on the universe, and a universe symmetry does not effect a genuine physical difference. Thus $\sigma_{\mathcal{S}}$ does not have DES. In particular, one cannot use it to effect a relational difference of the sort that is characteristic of Galileo's ship scenarios.

On the other hand, GW argue that the essence of Galileo's ship scenarios is captured by subsystem symmetries that are non-interior but are boundary-preserving. First, by non-interiority, such a symmetry $\sigma_{\mathcal{S}}$ does not, for all $s \in \mathcal{S}$, combine with $1_{\mathcal{\varepsilon}}$ to form a universe symmetry; (so it is not subject to the last paragraph's argument against exhibiting DES). Second, by boundary-preservingness, one can combine $\sigma_{\mathfrak{S}}$ with $1_{\mathcal{E}}$, because boundary-preserving subsystem symmetries will not change the dynamical compatibility relations between states in $\mathcal{S}$ and $\mathcal{E}$. Thus, it is possible to construct a composite transformation of the universe $\sigma_{\mathcal{S}} \sqcup 1_{\mathcal{E}}$ that is nonetheless not a symmetry of the universe, meaning that $\sigma_{S}$ can effect a physical difference and display DES. Furthermore, since each subsystem symmetry does not change the intrinsic properties of their respective subsystems, this physical difference will be purely relational, i.e. a Galileo's ship scenario.

It is straightforward to construct a Galileo's ship scenario for a scalar field $\phi$ with global $U(1)$ symmetry. By localizing this theory, we can obtain the model that GW call Klein-Gordon-Maxwell (KGM) electrodynamics ${ }^{24}$ This is their example of a non-interior, boundary-preserving subsystem symmetry in gauge theory. The Lagrangian density of this theory is:

$$
L=\left(D_{\mu} \phi\right)^{*} D^{\mu} \phi+m^{2} \phi^{*} \phi+F \wedge * F
$$

The space of states on the entire space-time manifold $M$ is $\mathcal{C} \equiv\{(A, \phi)\}$ (i.e. the space of field configurations satisfying the equations of motion) and gauge transformations act on the fields as follows:

$$
\begin{aligned}
& \phi \mapsto e^{-i q \chi} \phi, \\
& A \mapsto A+d \chi,
\end{aligned}
$$

where $\chi(t, x)$ is a smooth $\mathbb{R}$-valued function that parameterizes the gauge transformations.

GW take $\mathcal{S}$ to be the space of fields $\mathcal{C}_{S}$ on a compact space-time region $S$ with (connected) boundary $\partial S$, and they take $\mathcal{E}$ to be the space of fields on the space-time region $E=M-S$. They

\footnotetext{
${ }^{24}$ The KGM model is very similar to a $U(1)$ Yang-Mills-Higgs model, except with complex fields and a mass term.
} 
also interpret the condition that $\mathcal{S}$ is a dynamically isolated subsystem to mean that the fields $(A, \phi)$ vanish on $\partial S$.

Evidently, if we restrict our interest to gauge symmetries then the subsystem transformation of interest on $\mathcal{S}$ will be represented by the function $\chi_{S}: S \rightarrow \mathbb{R}$. GW claim that $\chi_{S}$ will represent a merely interior symmetry if it is zero on a small neighborhood of $\partial S$, meaning that the gauge symmetry $g=\exp (-i q \chi)$ tends smoothly to the identity near $\partial S{ }^{25}$

This claim may seem innocuous, but it in fact contains the assumption (Trivial $\Longrightarrow$ Interior): a gauge symmetry of a system $S$ is asymptotically trivial (in the sense that it tends to $I d$ on the $\partial S$ ) only if it is an interior symmetry ${ }^{26}$ In other words, the composability of $g$ with $1_{\varepsilon}$ (as a smooth function on the entire system) is taken to imply that $g \sqcup 1_{\varepsilon}$ is a universe symmetry. (Trivial $\Longrightarrow$ Interior) happens to be true in the above example, but in Section 4.2 we shall see that it is false in general.

GW further claim that gauge transformations can be non-interior and boundary-preserving. For instance, consider an 'asymptotic' gauge transformation parameterized by $\chi_{\infty}$, which tends towards a non-zero value on a neighborhood of $\partial S$. First, $\chi_{\infty}$ is non-interior since it is easy to find field configurations $s$ in $\mathcal{C}_{S}$ for which $s \sqcup e$ is dynamically possible, but not $\chi_{\infty}(s) \sqcup e{ }^{27}$ Second, in this 'dynamically isolated' scenario, $\chi_{\infty}$ is also boundary-preserving on any state $s$ that satisfies $\left.\phi\right|_{\partial S}=0$. This is because the environment states compatible with $s$, and their boundary class, are determined by the smooth gluing of $\left.\phi\right|_{E}$ with $\left.\phi\right|_{S}$ along $\partial S$ and given the boundary condition $\left.\phi\right|_{\partial S}=0$, none of this data is changed by an arbitrary gauge transformation on $S$.

Thus, we see that the example adduced by GW indeed meets all their desiderata for a Galileo's Ship scenario with respect to gauge symmetry (and more generally, local symmetry). Subject to the appropriate boundary conditions on $\partial S$, one can find an asymptotic subsystem transformation that changes the phase or the 'charge orientation' of the $\phi$ field within $S$ and thus creates a relational difference between the field configuration on $S$ and the field configuration on $E{ }^{28}$

\subsection{Collecting results, and the real issue}

Let us take stock and consider how the results of Section 3.2 relate to those of Section 3.1.

According to (GlobalInLocal) and (LocalizeEmpirical), there is a global symmetry contained in each gauge symmetry, and since the global symmetry can exhibit DES, so too can the gauge symmetry - by means of the global symmetry sitting inside it. GW's framework is supposed to give us an abstract way of picking out this DES-exhibiting global part of a gauge symmetry: we identify it as those subsystem symmetries which are non-interior and boundary-preserving. It then emerges that the global symmetries have the property of being asymptotically defined.

But as we saw in Section 3.1, it is in general false that a global symmetry is contained in a gauge symmetry, or that the definability of a charge from a global symmetry entails the definability of that same charge from the corresponding local symmetry. So for the general case, either (i) GW's characterization of DES-exhibiting symmetries is wrong; or (ii) their characterization does not in

\footnotetext{
${ }^{25}$ These functions have to tend smoothly towards their values on the boundary.

${ }^{26}$ Notice that this is roughly the same premise as 'Ext' in Friederich (2014).

${ }^{27}$ Just pick a field configuration that satisfies $\left.\phi\right|_{\partial S} \neq 0$.

${ }^{28}$ Having sketched the example, we can also sketch how GW could respond to Friederich $(2014)$ : they could say that they have no reason to accept his characterization of DES by means of the subsystem relation ' $\sim$ ', if this means anything other than empirical distinguishability with respect to an environment. Furthermore, if the latter is what is meant, then they have no reason to accept ' $\mathrm{MAH}$ ', because a relational difference between two subsystems need not imply a relational difference between the composite of the two and an environment.
} 
fact pick out a global symmetry that is embedded inside a local symmetry. In Section 4 we shall see that (ii) is indeed the case, because there are examples where the DES symmetry differs from the globalization of the gauge symmetry. In the atypical $U(1)$ example, the two happen to coincide, but this fact should not be taken as a guide to the general connection between gauge theories and Galileo's ship.

\section{Reconsidering the empirical significance of gauge symme- try}

GW are correct that the interior/non-interior distinction maps onto the non-DES/DES distinction ${ }^{29}$ However, this does not solve what I earlier (Section 1) called the logical puzzle about gauge, viz. how can the (Redundant) aspect of gauge transformations be squared with the claim that such transformations exhibit DES? Indeed, this puzzle would seem to be the source of the orthodox theorist's concerns that something must have gone awry in GW's analysis.

In Section 4.1, I will resolve this puzzle by sketching how constrained Hamiltonian analysis characterizes the (Redundant) gauge symmetries as a proper subgroup of the group of (Formal) gauge transformations. GW's view is thus able to avoid any contradiction with (Redundant) because the DES-exhibiting symmetries are given by quotienting out the group of (Redundant) gauge transformations from a larger group of gauge transformations which preserve appropriate boundary conditions.

Section 4.2 then presents a hierarchy of different groups of gauge transformations (including the group that characterizes (Redundant)) as subgroups of the group of (Formal) gauge transformations. It will be clear from this hierarchy that GW's suggestion that 'the DES-exhibiting symmetry of a gauge theory is the asymptotic globalization of its gauge symmetry' does not hold in general. This is shown by an example - the 't Hooft-Polyakov monopole - whose DES-exhibiting group is precisely not the globalization of its gauge symmetry.

\subsection{Gauge and redundancy}

What exactly is the formal characterization of a gauge symmetry that corresponds to (Redundant)? Many field theories are initially formulated in terms of a Lagrangian, and indeed considering the matter from this perspective may be particularly apt if one is interested in the spacetime geometry of field theory. However, the most straightforward approach is to consider the implications of constrained Hamiltonian analysis for a proper subsystem of the universe ${ }^{30}$

First, recall that our naive space of states is the space of field configurations $\mathcal{C}$ which satisfy the field equations and have finite energy. The evolution of a dynamical system is then given by a time-dependent curve $\gamma$ through $\mathcal{C}$. For instance, in the case of YMH theory, we have $t \mapsto \gamma(t)=$ $\left(A_{\mu}(t), \phi(t)\right) \in \mathcal{C}$. But there are redundancies in the YMH state space $\mathcal{C}$. For instance, one of the consequences of the equations of motion is Gauss' Law, which implies that the time-component $A_{0}$ of $A_{\mu}$ is determined by $A_{i}, \phi$, and their derivatives. Furthermore, it is easy to see that a set of initial data $(\gamma(0), \dot{\gamma}(0))$ does not determine a unique trajectory $\gamma(t)$ through $\mathcal{A}$. This is because gauge

\footnotetext{
${ }^{29}$ More precisely, the DES-exhibiting symmetries that are characteristic of Galileo's ship scenarios can be identified with the non-interior boundary-preserving symmetries.

${ }^{30}$ See Henneaux and Teitelboim (1992) for a detailed exposition of the constrained Hamiltonian formalism and its relation to quantization.
} 
transformations that are trivial at some initial time can become non-trivial at future times while remaining consistent with the equations of motions, and one can act on $\gamma(t)$ with these to produce a whole class of solutions to the equations of motion that have the same initial data.

We now sketch how the constrained Hamiltonian analysis of gauge systems eliminates such redundancies ${ }^{31}$ First, recall that a Lagrangian is defined on a space whose coordinates are the fields and their derivatives, i.e. $(q, \dot{q})$, where $q$ represents a field configuration. From the Legendre transformation $(q, \dot{q}) \mapsto(q, p)$, we obtain the Hamiltonian phase space, whose coordinates $q$ and $p$ represent fields and field momenta respectively. The crucial point here is that, in gauge theories, this map is not surjective; instead, the image of the map is a submanifold (called the constraint surface) of $P$ which is defined by the vanishing of a set of quantities $C_{j}(q, p)=0, \quad j=1,2,3, \ldots$, which are known as constraints.

We recall that the phase space $P$ has an algebraic structure, the Poisson bracket $\{\cdot, \cdot\}$, which is preserved by canonical transformations such as the dynamical evolution and the symmetries of the system. A function $f$ is said to generate a canonical transformation on a variable $h$ if its Poisson bracket with $h$ is equal to an infinitesimal transformation of $h$; exponentiating this infinitesimal transformation then results in a finite canonical transformation.

Let us now return to consider the constraints $C_{j}=0$. Since these do not vanish everywhere on $P$, there exist functions $f$ whose Poisson bracket with $C_{j}$ does not vanish on the constraint surface; in other words, they generate canonical transformations that move points out of the constraint surface. Because the equation $C_{j}=0$ does not remain valid if we take Poisson brackets $\{f, \cdot\}$ on both sides of it, we say that it vanishes weakly, and denote this by $C_{j} \approx 0$. This is a very fruitful approach to analyzing the dynamics of a physical system. For instance, one can show that in order for the dynamics to be consistent the Poisson brackets of the constraints $C_{j}$ and the Hamiltonian $H$ are required to vanish weakly. This requirement may in turn lead to other constraints, and by iterating the process of taking Poisson brackets in this way, one obtains the complete set of constraints that is required for the consistency of the dynamics - let us continue to denote this set by $\left\{C_{k} \approx 0\right\}$.

It is conventional wisdom in constrained Hamiltonian dynamics that the (Redundant) gauge transformations are generated by the first-class constraints, i.e. those constraints $K_{l} \approx 0$ for which $\left\{K_{l}, C_{k}\right\} \approx 0{ }^{32}$ In the YMH case there are two first-class constraints: the momentum constraint $\pi^{0} \approx 0$, and the Gauss' Law constraint $D_{i} \pi^{i} \approx 0$, where $\pi^{\mu}=F^{\mu 0}$.

The formal elimination of the redundancies can now be carried out in the Hamiltonian approach. Just as in Section 2.2, one introduces an equivalence relation between points connected by transformations generated by the first class constraints - the equivalence classes are the orbits of the action of these transformations. Physical (often called: gauge-invariant) quantities are then taken to be the functions which are constant on these orbits. The space of orbits is thus free of redundancies and has a deterministic initial value problem.

Among other things, the constrained Hamiltonian analysis offers a framework within which one can give a formal characterization of (Redundant) gauge transformations. In particular, Balachandran (1994) has used it to argue that these transformations must take certain asymptotic values on the boundary of a subsystem, on pain of the Poisson brackets between first class constraints and physical quantities of interest being ill-defined. For instance, in the case of YMH theory, the argument runs as follows: smear the Gauss constraint with a test function in order to obtain a

\footnotetext{
${ }^{31}$ Our sketch roughly follows Balachandran $(1994)$, but there are many good sources: see e.g. Henneaux and Teitelboim (1992) for a magisterial overview.

${ }^{32}$ See Section 1.2 of Henneaux and Teitelboim (1992) for a justification of the conventional wisdom, and see Pitts (2014) for an important refinement.
} 
generator of gauge transformations (which we can identify by the action of its Poisson brackets on $A_{i}$ and $E_{i}$.) Next, evaluate the Poisson bracket of the smeared Gauss constraint with a physical quantity like $J_{i}$, the generator of rotations - one sees that there are two different ways of evaluating the Poisson bracket (based on two possible orders of integration), and the answers do not agree unless asymptotic conditions are imposed on the gauge transformations. Balachandran showed that the simplest such condition is ${ }^{33}$

$$
g(x) \rightarrow \mathrm{Id} \quad \text { as } \quad|x| \rightarrow \infty .
$$

Furthermore, the transformations generated by this constraint can be smoothly deformed to the identity Id $\in G$ - transformations that have this property are called 'topologically trivial' or 'small' 34 By contrast, the 'large' gauge transformations are defined as the ones that cannot be smoothly deformed to the identity. We will use $\mathcal{G}_{0}^{\infty}$ to denote the group of (Redundant) gauge transformations, i.e. those small gauge transformations which satisfy (9).

Let us pause to consider the morals of the constrained Hamiltonian analysis for our topic. As we noted in Section 2.1 above, it is widely acknowledged - on philosophical grounds - that every symmetry transformation (and not just gauge transformations) of the universe connects redundant descriptions. On the other hand, when it comes to proper subsystems of the universe, many share the intuition that there is a fundamental difference between global transformations and gauge transformations: the former describe operations that can (directly or indirectly) be performed on the subsystem (and constitute a physical change in relation to its environment), whereas the latter are just mathematical artifacts of redundant descriptions of the subsystem.

The constrained Hamiltonian analysis of 'gauge' teaches us how to square these two ideas. First, it uses physical reasoning (in the form of the consistency of Poisson brackets) to identify $\mathcal{G}_{0}^{\infty}$ as the subgroup of (Formal) gauge transformations that is responsible for mathematical redundancy. Second, it is natural to introduce the assumption that since elements of $\mathcal{G}_{0}^{\infty}$ are deformable into the identity, they are interior symmetries in GW's sense. In other words, they are subsystem symmetries that extend to symmetries of the universe: one thereby squares the two prima facie distinct notions of 'redundancy through being a symmetry of the universe' and 'redundancy through being a gauge symmetry'. Indeed, one might try to use analyze the latter type of redundancy in terms of the former, i.e. (Redundant) gauge symmetries are redundant in virtue of the fact that they are a restriction of universe symmetries ${ }^{35}$

To recapitulate: the constrained Hamiltonian formalism shows that given some proper subsystem, the specific type of redundancy that comes from the notion of 'gauge transformation' is not captured by the (Formal) group of gauge transformations $\mathcal{G}$, but rather the restriction to its subgroup $\mathcal{G}_{0}^{\infty}$. In the next section, we will see that $\mathcal{G}_{0}^{\infty}$ is the smallest of three physically relevant subgroups of $\mathcal{G}$, each of which plays an important conceptual role in interpreting gauge symmetry.

\subsection{Different groups of gauge transformations}

In what follows, we will only be interested in time-independent solutions to YMH theory (since the standard strategy for constructing solitons is to find such solutions and then boost them in order

\footnotetext{
${ }^{33}$ See pp. 21-22 of Balachandran (1994).

${ }^{34}$ This property is not a deep physical fact, but rather a formal consequence of how one constructs such continuous symmetry transformations, viz. by exponentiating generators. It follows that a limitation of the constrained Hamiltonian framework is that it does not have much to say about discrete symmetries.

${ }^{35} \mathrm{I}$ do not however make any claims about whether this is the most illuminating way in which to understand gauge symmetry.
} 
to introduce time-dependence). As such, gauge transformations will be maps from space (which we take to be $\mathbb{R}^{3}$ ) to a gauge group $G$.

The (Formal) group of gauge transformations that we first naively introduced in Section 2.2 is simply

$$
\mathcal{G}:=\left\{g: \mathbb{R}^{3} \rightarrow G\right\},
$$

without any asymptotic conditions imposed. Next, and in keeping with the insights of the previous section, let us define the asymptotically trivial group of gauge transformations

$$
\mathcal{G}^{\infty}:=\left\{g: \mathbb{R}^{3} \rightarrow G \mid g(x) \rightarrow \text { Id } \quad \text { when }|x| \rightarrow \infty\right\}
$$

As previously stated, constrained Hamiltonian analysis shows us that the (Redundant) group of gauge transformations is $\mathcal{G}_{0}^{\infty}$, i.e. the subgroup of $\mathcal{G}^{\infty}$ that is smoothly connected to the identity. We are now in a position to see why GW's claim that (Trivial $\Longrightarrow$ Interior) is false: in some cases, it can turn out that $\mathcal{G}^{\infty} \equiv \mathcal{G}_{0}^{\infty}$, but in general (e.g. for the $S U(2)$ monopoles discussed below) asymptotically trivial transformations can turn out to be topologically non-trivial; thus the two groups cannot be identified.

In fact, these three groups do not exhaust the set of physically relevant distinctions that one might draw with respect to 'the group of gauge transformations', because there is a fourth possibility that was already anticipated in our discussion of KGM electrodynamics at the end of Section 3.2. In the KGM example, the boundary condition of the subsystem $\mathcal{S}$ was specified by the requirement that the field $\phi$ vanish in a neighborhood of the (space-time) boundary $\partial S$ of $S$. In general, such boundary conditions (in conjunction with the initial conditions and equations of motion) need to be laid down in order to specify a well-defined dynamical system, and should be treated as part of the framework of the physical problem that one wants to solve. Thus, the dynamical evolution and the symmetry transformations of the system should leave such boundary conditions invariant. In the KGM case, it was trivial to satisfy the requirement that a gauge transformation leave the boundary condition $\left.\phi\right|_{\partial S}=0$ invariant. But it is entirely conceivable that this requirement will not be so trivially satisfied when one is dealing with more complex theories, in which case we are led to schematically define our fourth group of gauge transformations: the invariance group $\mathcal{G}_{I}$, which leaves invariant the boundary conditions of the fields, whatever they might be.

Notice that these four groups form a hierarchy of subgroups:

$$
\mathcal{G}_{0}^{\infty} \subset \mathcal{G}^{\infty} \subset \mathcal{G}_{I} \subset \mathcal{G} .
$$

We can now see the punchline. The group of (subsystem) gauge symmetries that is a candidate for exhibiting DES must satisfy two desiderata: (1) it must leave the boundary conditions of the fields fixed (otherwise the transformation would change the type of field configuration whose properties were being studied); and (2), it must exclude the interior subsystem symmetries, i.e. the (Redundant) part of the gauge transformations, which cannot exhibit DES. We need the structure of $\mathcal{G}_{I}$ to satisfy (1), but we also need to quotient this by $\mathcal{G}_{0}^{\infty}$ to satisfy (2). Thus: the DES-exhibiting group of symmetries must be the quotient group

$$
\mathcal{G}_{D E S}:=\mathcal{G}_{I} / \mathcal{G}_{0}^{\infty} .
$$

An informal version of this result was already present in (Greaves and Wallace, 2014, p. 29), but a more careful statement will allow us to see why one should refrain from identifying the DESexhibiting part of a gauge symmetry with its globalization. 
In order to forestall a possible misunderstanding, it is instructive to contrast this result with the case of global symmetries, which can exhibit DES (as in the original Galileo's ship scenario). In particular, one might wonder whether (i) the idea that global symmetries, which both relate redundant descriptions (when they are symmetries of the universe) and also have the potential to exhibit DES conflicts with (ii) the idea that (Redundant) gauge transformations need to be eliminated in order to obtain a DES-exhibiting group of symmetries. The answer is that there is no such conflict, because (non-trivial) global symmetries can only exhibit DES when they are symmetries of proper subsystems, and in this context, they do not extend to symmetries of the universe - thus there are no such redundancies to be eliminated. By contrast, the notion of a gauge symmetry allows for a rich structure of non-trivial subsystem transformations which are also symmetries of the universe - this is precisely the (Redundant) part of $\mathcal{G}$ that does not exhibit DES.

Let us now revisit the KGM example with the tools that we have developed in this section. In that case, $G_{I}$ is the group of gauge transformations that asymptotically tend towards a constant value on $\partial S . \mathcal{G}_{D E S}$ is easily seen to be $U(1)$ by performing the quotient group computation in (13), which might tempt one to generally identify the DES-exhibiting symmetry of a gauge theory with the globalization of the theory's (Formal) gauge symmetry on the boundary $\partial S$. But this would be a mistake: we can see from (13) that such an identification would be false if $\mathcal{G}_{I}$ had a different structure. Such is the case with the 't Hooft-Polyakov monopole, whose analysis we consider in Appendix A.

\section{Concluding morals}

Let us sum up. Against the philosophical orthodoxy, GW and I claim that gauge symmetry can indeed exhibit DES in a way that is characteristic of Galileo's ship scenarios, viz. a gauge symmetry acting on a subsystem can indeed effect a genuine relational difference between the subsystem and its environment. (Those who remain unconvinced should also consider the argument of de Haro et al. (2015), where we apply the AdS/CFT duality to show that a gauge transformation in the bulk theory is translated into a global conformal symmetry in the boundary theory.)

Part of GW's motivation stems from the intuitively plausible idea that global symmetry is contained in (i.e. 'the globalization of') gauge symmetry, and since global symmetry can exhibit DES, so too can gauge symmetry. However, we have seen that this picture, though suggestive, is incorrect for general gauge theories. The correct view is that DES symmetries are determined by the conjunction of (i) boundary conditions, and (ii) the formal properties of gauge symmetry upon quotienting out by the redundancy-generating part of the symmetry. This allows for scenarios (e.g. solitonic systems) where the DES symmetry is precisely not a globalization of the gauge symmetry.

Finally, I list three issues that merit further philosophical attention. First, it would be nice to have a general account of how the problems with defining charge in general gauge theories relate to the empirical content of a gauge theory. Second, we have noted that 'large' asymptotically trivial gauge transformations need not be interior - how is this topological non-triviality relevant to DES and the set of universe symmetries: ${ }^{36}$ Third, we have shown that the preservation of empirical content in the process of localization cannot in general be explained by (GlobalInLocal); how then is such content (if any) preserved?

\footnotetext{
${ }^{36}$ See also the treatment of dyon solutions in Witten $(1979)$, which seems to treat $\mathcal{G}^{\infty}$ as interior by adding a topological term to the Lagrangian.
} 


\section{References}

Abbott, L. and Deser, S. (1982). Charge definition in non-abelian gauge theories. Physics Letters, 116B.

Balachandran, A. (1994). Gauge symmetries, topology and quantization. AIP Conf.Proc., 317:1-81.

Barnich, G. and Brandt, F. (2002). Covariant theory of asymptotic symmetries, conservation laws and central charges. Nuclear Physics B, 633:3-82.

Belot, G. (2011). Symmetry and equivalence. Proceedings of PSA, 22.

Brading, K. and Brown, H. (2004). Are gauge symmetry transformations observable? British Journal for the Philosophy of Science, 55 (4):645-65.

Brading, K. and Brown, H. R. (2000). Noether's Theorems and Gauge Symmetries. ArXiv High Energy Physics - Theory e-prints, hep-th/0009058.

Brown, H. R. and Sypel, R. (1995). On the meaning of the relativity principle and other symmetries. International Studies in the Philosophy of Science, 9:23553.

Budden, T. (1997). Galileo's ship and spacetime symmetry. British Jounal for the Philosophy of Science, 48:483-516.

de Haro, S., Teh, N., and Butterfield, J. (2015). Comparing dualities and gauge symmetries. in submission.

Friederich, S. (2014). Symmetry, empirical equivalence, and identity. British Jounal for the Philosophy of Science.

Galileo (1953). Dialogues Concerning the Two Chief World Systems. University of California Press.

Greaves, H. and Wallace, D. (2014). Empirical consequences of symmetries. British Journal for the Philosophy of Science, 65:59-89.

Healey, R. (2009). Perfect symmetries. British Journal for the Philosophy of Science, 60:697-720.

Henneaux and Teitelboim (1992). Quantization of Gauge Systems. PUP.

Kosso, P. (2000). The empirical status of symmetries in physics. BJPS, 51:81-98.

Nussinov, Z., Batista, C. D., and Fradkin, E. (2006). Intermediate Symmetries in Electronic Systems:. Dimensional Reduction, Order Out of Disorder, Dualities, and Fractionalization. International Journal of Modern Physics B, 20:5239-5249.

Olver, P. (1993). Applications of Lie Groups to Differential Equations. Springer.

Pitts, J. B. (2014). A First Class Constraint Generates Not a Gauge Transformation, But a Bad Physical Change: The Case of Electromagnetism. Annals Phys., 351:382-406.

Witten, E. (1979). Dyons of charge e $\theta / 2 \pi$. Physics Letters B, 86:283-287. 